G65(P)

\section{RISK OF INVASIVE PNEUMOCOCCAL DISEASE IN CHILDREN WITH SICKLE CELL DISEASE IN THE ERA OF PNEUMOCOCCAL CONJUGATE VACCINES: A SYSTEMATIC REVIEW OF THE LITERATURE}

${ }^{1} \mathrm{G}$ Oligbu, ${ }^{2} \mathrm{~L}$ Pay, ${ }^{2} \mathrm{M}$ Fallaha, ${ }^{3} \mathrm{O}$ Oligbu, ${ }^{4} \mathrm{U}$ Nwaomu. ${ }^{1}$ Paediatric Infectious Diseases Research Group, St George's University of London, UK; ${ }^{2}$ Department of Paediatrics, Imperial College London, UK; ${ }^{3}$ Accident and Emergency Department, Queen Elizabeth Hospital, Woolwich, London, UK; ${ }^{4}$ Department of Paediatrics, Royal Derby Hospital, Derby, UK

\subsection{6/archdischild-2018-rcpch.63}

Introduction Invasive pneumococcal disease (IPD) is the leading cause of morbidity and mortality in children with sickle cell disease (SCD). 7-valent pneumococcal conjugate vaccine (PCV7) was first introduced in 2000 in US and at various times in other countries. It was replaced by 13 -valent pneumococcal conjugate vaccines (PCV13) in 2010. This PCV are highly effective in preventing IPD in children with SCD. The risk of IPD has not been systematically assessed in children with SCD since the introduction of PCV.

Methods We undertook a systematic review of the English literature published from 2000 to October 2017 to evaluate the risk factors, serotype distribution, clinical presentation and outcomes of IPD in children with sickle cell disease. Data sources included MEDLINE, EMBASE Cochrane library, and references within identified articles.

Results We identified 475 potential studies, of which 66 were duplicates. A further 299 were excluded on the basis of title and abstracts and another 94 studies did not meet eligibility criteria on full-text screening. We included 16 publications involving 11383 children less than 21 years-olds with SCD. A total of $161(1.4 \%)$ IPD was identified: 147 homozygote for haemoglobin S (HbSS), 10 double heterozygote for haemoglobin S and C (HbSC) and 4 others. Among the nine studies reporting clinical presentation, septicaemia was the commonest $(n=69 / 108 ; 63.8 \%)$ followed by pneumonia $(n=20 / 108$; $18.5 \%)$. The serotypes associated with IPD in SCD were mainly non-PCV13 ( $\mathrm{n}=117 / 133 ; 88 \%)$, of which more than a quarter was due to serogroup $15 \mathrm{~A} / \mathrm{B} / \mathrm{C} \quad(\mathrm{n}=34 / 117 ; 29 \%$,). Serotype 23 F $(n=6 / 16,37.5 \%)$ and 7 F $(n=4 / 16 ; 25 \%)$ were the main vaccine serotypes. Majority were sensitive to penicillin and ceftriaxone. The crude case fatality rate was $11.2 \%$ $(\mathrm{n}=18 / 161)$.

Conclusions and clinical implications This report demonstrates the effectiveness of conjugate vaccines to reduce the rate of IPD in children with SCD. However, these children remain at increased risk and are also more likely to die of their infection compared to their peers without SCD. Most IPD cases are now due to serotypes not covered by PCV13. Therefore better prevention strategies are needed to reduce the overall burden IPD in children with SCD.

\section{G66(P) REDUCED RATES OF JUVENILE ONSET RECURRENT RESPIRATORY PAPILLOMATOSIS IN AUSTRALIA AFTER IMPLEMENTATION OF A NATIONAL HPV VACCINATION PROGRAM}

1) Brotherton, 2,3Y Zurynski, ${ }^{4} \mathrm{~A}$ Cheng, ${ }^{5} \mathrm{SM}$ Garland, ${ }^{3,4,6} \mathrm{R}$ Booy, ${ }^{2,3,4} \mathrm{EJ}$ Elliott, ${ }^{7}$ D Novakovic. 'National HPV Vaccination Program Register, VCS, East Melbourne, Victoria, Australia; ${ }^{2}$ Australian Paediatric Surveillance Unit, Kids Research Institute, Sydney, Australia; ${ }^{3}$ Discipline of Child and Adolescent Health, The University of Sydney, Sydney, Australia; ${ }^{4}$ Sydney Children's Hospitals Network, the Children's Hospital Westmead, Sydney, Australia; ${ }^{5} 12$ Royal Women's Hospital, Department of Microbiology and Infectious Diseases, Parkville, and Murdoch Childrens Research Institute, Infection and Immunity Theme, Melbourne, Australia; ${ }^{6}$ National Centre for Immunisation Research and Surveillance, Kids Research Institute, Sydney, Australia; 'University of Sydney Medical School, University of Sydney, Sydney, Australia

\subsection{6/archdischild-2018-rcpch.64}

Aims Juvenile onset Recurrent Respiratory Papillomatosis (JoRRP) is a rare chronic disease caused by human papillomavirus (HPV) types 6 and 11. Children with RRP require multiple surgical interventions. Tracheostomy may be needed and sometimes the disease is fatal. These infections are now preventable through HPV vaccination. Following an extensive quadrivalent HPV vaccine catch-up program for females aged 12-26 years in 2007-2009, in Australia, we aimed to monitor the changes in incidence and demographics of JoRRP over time.

Methods The Australian Paediatric Surveillance Unit (APSU) conducted national surveillance for JRRoP using its well established reporting system. In addition to the $\sim 1450$ paediatricians who report to the APSU each month, paediatric otorhinolaryngologists were also enrolled in the APSU and offered HPV typing. We report findings for the five-year period to end 2016.

Results The average annual incidence rate was 0.0715 per 100000 children aged $<16$ years. The largest number of cases was reported in the first year, with decreasing annual frequency thereafter. The rate declined significantly from 0.163 per 100000 in 2012 to 0.024 per 100000 in 2016 $(\mathrm{p}=0.034)$. Among the 15 incident cases $60 \%$ male, $60 \%$ were first born children and 13 (87\%) were born vaginally. None of the mothers of these children had received the HPV vaccine before pregnancy, and $3(20 \%)$ of the mothers had a history of genital warts. Seven genotyped cases were positive for HPV including 4 that were HPV6 positive and 3 that were HPV11 positive.

Conclusion To our knowledge this is the first report internationally documenting decline in JoRRP incidence in children following a quadrivalent HPV vaccination program. 


\section{British Association of General Paediatrics and Paediatric Mental Health Association}

\section{G67 THE COLLABORATIVE DEVELOPMENT OF A REGIONAL PAEDIATRIC SEPSIS SCREENING TOOL}

${ }^{1} \mathrm{H}$ Rutkowska, ${ }^{2} \mathrm{~J}$ Baird, ${ }^{3} \mathrm{P}$ Parslow, ${ }^{4} \mathrm{~W}$ Portas, ${ }^{4} \mathrm{G}$ Paul, ${ }^{1} \mathrm{~J}$ Mills, ${ }^{5} \mathrm{~S}$ Nicholls, ${ }^{5} \mathrm{M}$ Linney. ${ }^{1}$ Department of Paediatrics, University Hospital Southampton NHS Foundation Trust, Southampton, UK; ${ }^{2}$ Department of Paediatrics, Salisbury NHS Foundation Trust, Salisbury, UK; ${ }^{3}$ Department of Paediatrics, Dorest County Hospital NHS Foundation Trust, Dorchester, UK; ${ }^{4}$ University of Southampton Faculty of Medicine, University Hospital Southampton NHS Foundation Trust, Southampton, UK; ${ }^{5}$ Department of Paediatrics, Western Sussex Hospitals NHS Foundation Trust, Chichester, UK

\subsection{6/archdischild-2018-rcpch.65}

Aims Our Paediatric Sepsis Working Group was formed as a result of a number of timely drivers: local learning from clinical cases, the Government Sepsis CQUIN and imminent publication of NICE guidance on sepsis. Our local Academic Health Sciences Network held a sepsis-focussed breakthrough Collaborative and we agreed to join as a regional group. This was based on our assumption that the well-established Regional Paediatric Critical Care Network would be in an excellent position to successfully deliver regional system change.

Method Over 14 PDSA cycles, a regional Paediatric Sepsis Screening Tool was developed and tested by members of the multi-professional team across the region, gaining point-of-care feedback at each stage and analysing collective data. The Tool was piloted in 5 hospitals within the network before being rolled out region-wide. The tool has also been through two large audits, comprising of the study of 930 acute admission records. User feedback was collected throughout.

Results Our newly developed Regional Paediatric Sepsis Screening Tool (RPSST) reliably detected all blood culture positive septic patients and those with severe bacterial infections causing physical compromise. User feedback has proven that it is quick and easy to use. The RPSST was also shown to trigger $50 \%$ less patients than the current NICE guidance recommends for immediate senior review. There was concern that the introduction of a trigger tool may adversely affect our antibiotic prescribing. Analysis of our local antibiotic prescribing data has not shown an increase in Ceftriaxone prescribing since the Tool's introduction (static at 6\%), demonstrating that the new RPSST is being used effectively within the clinical context.

Conclusions This Tool has been successfully incorporated into our acute paperwork across the region, enabling Trusts to address the CQUIN targets whilst highlighting this important clinical problem. The Regional PSST compares favourably with the NICE guidance. Collaborative working has reduced the burden of individual working and enabled wider regional engagement, sharing knowledge and expertise and reducing variation in practice. This is supported by the PIER network locally (www.piernetwork.org). We would encourage other regions to explore collaborative working to improve outcomes for patients.

\section{G68} MENTAL HEALTH ADMISSIONS TO AN ACUTE PAEDIATRIC WARD

${ }^{1} \mathrm{~K}$ Pierson, ${ }^{1} \mathrm{~A}$ Merino Elia, ${ }^{1} \mathrm{~F}$ Seregni, I Takon, ${ }^{2} \mathrm{~T}$ Govender. ${ }^{1}$ Paediatrics, East and North Hertfordshire Trust, Stevenage, UK; ${ }^{2}$ Child and Adolescent Mental Health Services, Hertfordshire Partnership University NHS Foundation Trust, Hatfield, UK

\subsection{6/archdischild-2018-rcpch.66}

Aims $10 \%$ of children and young people in the UK have a diagnosable mental health problem. NICE Guidelines recommend that Acute Paediatric Services provide a place of safety for young people who present to A and E, while a mental health assessment takes place. The aim of this study was to evaluate the service and safety of children and young people with mental health problems admitted to our acute paediatric ward.

Methods We carried out a retrospective analysis of all CAMHS patients admitted to our acute paediatric ward, over a 3 month period. The study looked at reasons for presentation and admission, demographics, documentation of risk assessment, waiting time for an initial CAMHS assessment, presence of a one to one Registered Mental Health Nurse (RMN), documentation of observations, recurrence of admission for similar problem, length of stay, and outcome/follow up at discharge.

Results Patients were aged 11 to 17 years with $82 \%$ being female. Less than 30\% had risk assessments and none had RMNs. $85 \%$ were admitted awaiting CAMHS assessment, of which $79 \%$ were previously known to CAMHS and 50\% had previous mental health admissions to our paediatric ward. Not all patients had regular observations, with 33\% having only an initial set of observations carried out. All except one had a CAMHS assessment within 24 hours of presentation and $74 \%$ of these were discharged within 24 hours. Three patients were transferred to an inpatient mental health unit.

Conclusions Children and young people with mental health problems constitute a significant percentage of admissions to paediatrics. Many of them are recurrent attenders known to CAMHS. Most of the patients were seen by CAMHS within 24 hours as per NICE guidelines. However, with poor documentation of risk assessments, no RMN presence, and scattered observations, there is a need for improvement to the safety of mental health admissions to acute paediatric wards.

\section{G69 HOW DO COMMUNITY PHARMACIES SUPPORT CHILDREN WITH MINOR ILLNESS?}

${ }^{1} \mathrm{M}$ Blair, ${ }^{1} \mathrm{G}$ Oligbu, ${ }^{2} \mathrm{O}$ El Tokhy, ${ }^{3} \mathrm{M}$ Levitan, ${ }^{4} \mathrm{P}$ Goldstone, ${ }^{4} \mathrm{P}$ Lathlean. ${ }^{1}$ Paediatrics and Child Health, Imperial College, London, UK; ${ }^{2}$ Imperial College, London, UK; ${ }^{3}$ Middlesex Pharmacy Group, LPC, London, UK; ${ }^{4}$ Enfield Primary Care, Clinical Commissioning Group, London, UK

\subsection{6/archdischild-2018-rcpch.67}

Background Many parents are using ED services as a first point of contact. A large proportion of the attendances are low acuity. Greater use of community pharmacy services for minor ailments could help relieve pressure on healthcare 